\title{
Preemptive Ketamine in Abdominal Hysterectomy Patients, Does the Route of Administration Affect Analgesia and Hemodynamics? A Prospective Randomized Controlled Trial Mohamed Adel Aboelela \\ Department of Anesthesia and Surgical Intensive Care, Mansoura University,Egypt. Corresponding author:Mohamed adel aboelela,email:aboelela_mohamed@yahoo.com, ORCID: https://orcid.org/0000-0001-8019-0243,Mobile: 01061546753
}

\section{ABSTRACT}

Introduction: Hysterectomy is a common procedure and postoperative pain is a considerable problem. Preemptive analgesia includes analgesic administration prior to surgical stimulus. Ketamine is a short acting anesthetic drug with analgesic and anti-inflammatory properties, reducing central sensitization of pain pathway and hyperalgesia.

Objectives: This study aimed to assess the efficacy of preemptive use of ketamine as an analgesic drug, either by intravenous route or by subcutaneous infiltration at the incisional site, regarding perioperative hemodynamics, postoperative pain and gastrointestinal irritation symptoms.

Patients and methods: 60 patients were enrolled in this study, divided into two equal groups (30 each) according to route of preemptive ketamine administration. Group (V) received $0.5 \mathrm{mg} \mathrm{kg}^{-1}$ intravenously while group (S) received $0.5 \mathrm{mg} . \mathrm{kg}^{-1}$ subcutaneously at incisional site. Postoperative pain assessment for $24 \mathrm{H}$. using visual analogue score (VAS) was adapted as primary outcome objective, while secondary outcomes included hemodynamic parameter and postoperative nausea and vomiting (PONV). Results: Data were analysed. VAS score showed significant difference among studied groups in early 2 postoperative hours. $\mathrm{VAS}^{0}\left(\mathrm{~V} 2.40 \pm 0.62\right.$, S 1.96 $\pm 0.92, \mathrm{P} 0.038$ ), $\mathrm{VAS}^{1}$ (V 3.96 \pm 1.21 , $\mathrm{S} 2.86 \pm 0.89, \mathrm{P} 0.001$ ), $\mathrm{VAS}^{2}$ (V 4.00 $\pm 0.94, \mathrm{~S} 3.33 \pm 0.88, \mathrm{P} 0.007$ ). Also, first time to pethidine intake (V $1.50 \pm 0.93 \mathrm{H}$, $\mathrm{S} 2.36 \pm 1.27 \mathrm{H}, \mathrm{P} 0.004)$. Other study objectives were comparable.

Conclusion: Preemptive use of ketamine with subcutaneous infiltration at incisional site in total abdominal hysterectomy patients provided better analgesia in early postoperative hours rather than intravenous route with comparable hemodynamic parameter and side effects.

Keywords: Hemodynamics, Hysterectomy, Pain, Preemptive ketamine, VAS.

\section{INTRODUCTION}

Hysterectomy is a frequent gynecological procedure with highest rate observed among women aging 40 to 49 years old. In most cases, it is used to treat a certain types of gynecological diseases not responding well to medical treatments such as pelvic pain, intense or irregular monthly bleeding, prolapse and uterine cancers ${ }^{(1)}$.

Post hysterectomy pain is one of the most common complaints among such patients, leading to decrease patients satisfaction, delayed mobilization, cardiopulmonary dysfunction, increase hospital stay and increased morbidity and mortality risk ${ }^{(2,3)}$.

Analgesia is an important component of anesthetic plan. Effective management of postoperative pain is expected to improve perioperative quality of recovery (4). Preemptive analgesia consists of administration of the analgesic drugs prior to surgery. It acts through reducing nociceptive input, rising of the nociception threshold, and reducing activation of nociceptive receptors prior to making the surgical incision ${ }^{(5)}$. Preemptive analgesia provides better pain management, reducing rescue analgesia consumption and improving patient's satisfaction ${ }^{(6)}$.

Ketamine is a short acting anesthetic drug that has analgesic properties at sub-anesthetic doses as $\mathrm{N}$ methyl D-aspartate (NMDA) receptor antagonist with anti-inflammatory properties ${ }^{(7)}$. Ketamine produces central desensitization of pain pathway, reducing central hyperalgesia specially when used preemptively ${ }^{(8)}$. In this trial, we used preemptive ketamine and compared two different route of administration, either pre-incisional subcutaneous infiltration or preincisional intravenous administration. Postoperative pain assessment using visual analogue score (VAS) was adapted as primary outcome objective, while secondary outcomes included hemodynamics heart rate (HR) and mean blood pressure (MBP), PONV and rescue analgesia consumption (total dose and first time to analgesia request).

\section{PATIENTS AND METHODS}

This study was conducted at Mansoura University Hospital, sixty patients were enrolled in this study, which adheres to the applicable CONSORT guidelines. Ethical approval:

This study was approved by the Institutional Review Board, Mansoura Faculty of Medicine (IRB \# R.20.06.911, July 8-2020), and informed consent was obtained from all subjects participating in the trial. Before patient enrolment, the trial was registered in the Pan African Clinical Trial Registry (PACTR202007562388257, date of registration: July 17-2020). Included females were ASA I or II, aging 18-65 years, scheduled for total abdominal hysterectomy.

Exclusion criteria: Patient refusal to participate, having allergy to used drug, addiction, psychological troubles, uncontrolled hypertension or cardiac disorder, 
altered liver or kidney functions and complicated surgery.

Random number generator with closed envelope technique, allocated the patients into two equal groups based on the route of administration (Figure 1); subcutaneous ketamine group ( $\mathrm{S}$ group) $(\mathrm{n}=30)$, and intravenous ketamine group ( $\mathrm{V}$ group) $(\mathrm{n}=30)$.

According to our policy, patients had preoperative visit for anesthetic evaluation, technique explanation and getting the informed consent. Patients were assessed clinically, documented medical and surgical history were taken, investigation including (ECG, CBC, LFTs, RFTs, Coagulation profile) were done. In the operative suite, patients were connected to monitor (General electric - Datex B850, USA) for monitoring ECG, NIBP, oxygen saturation and recording basal data. A 18 $G$ venous catheter was inserted in the non-dominant hand. Premedication included pantoprazole (Zurcal 40 $\mathrm{mg}$, AUG pharma, Spain) and $3 \mathrm{mg}$ midazolam (Midathetic, Amoun pharmaceuticals, Egypt). At the operating room, patients were connected to anesthesia monitor for monitoring of ECG, NIBP, End tidal $\mathrm{CO}_{2}$ and oxygen saturation. Anesthesia was induced using propofol 1-2 mg.kg-1 (diprivan, Fresenius KABI, Egypt), fentanyl $1 \mu \cdot \mathrm{kg}^{-1}$ (fentanyl Hameln, Hameln pharmaceuticals, Germany) and atracurium $0.6 \mathrm{mg}$. $\mathrm{kg}^{-}$ 1 (Atrabesylate, Egypharm, Egypt). A proper sized endotracheal tube was inserted and fixed in place after confirmation of correct position by chest auscultation and capnogram. Patients were ventilated using (GE Datex-Ohmeda Aisys (USA) ventilator) with volume controlled ventilation (VCV) mode to keep $\mathrm{EtCO}_{2} 35 \pm 2$ $\mathrm{mmHg}$. Anesthesia was maintained using sevoflurane (sevoran, abbott, Egypt) $1-2 \%$ in $40 \%$ oxygen air gas mixture, top up dose of atracurium was given on needs. Before starting surgery, study protocol was initiated according to randomization number received in a closed envelop with patients file. Anesthesia technician prepared two covered syringes labelled by randomization numbers for each patient. The first syringe was $10 \mathrm{ml}$ for intravenous injection, contained $0.5 \mathrm{mg} \cdot \mathrm{kg}^{-1}$ ketamine in $10 \mathrm{ml}$ saline for $\mathrm{V}$ group or 10 $\mathrm{ml}$ saline only as placebo in $\mathrm{S}$ group. The second syringe was $20 \mathrm{ml}$ for subcutaneous infiltration, contained $0.5 \mathrm{mg} \cdot \mathrm{kg}^{-1}$ ketamine in $20 \mathrm{ml}$ saline for $\mathrm{S}$ group or $20 \mathrm{ml}$ saline only as placebo in $\mathrm{V}$ group. Both syringes $(10 \mathrm{ml}, 20 \mathrm{ml})$ were given to researcher anesthesiologist for drug injection $10 \mathrm{~min}$ before starting surgery while blinded of drug composition of both syringes. Another 18 or 20 G IV line was inserted to infuse ringer acetate with a rate $4 \mathrm{ml} . \mathrm{kg}^{-1} \cdot \mathrm{h}^{-1}$ and other fluids or blood product according to clinical transfusion needs during surgery. Also, a suitable size urinary catheter was inserted before starting surgery. 1 gm of paracetamol (perfelgan, Bristol Mayers Squibb, USA) was infused IV in both groups as a part of multimodal analgesia technique before skin incision. Surgery started with pfannenstiel incision, intraoperative hemodynamics monitoring and management were done by the researcher anesthesiologist responsible for data recording intraoperatively and in Post-Anesthesia Care Unit (PACU). After completion of surgical procedure, recovery of muscle relaxation were done using neostigmine $0.05 \mathrm{mg} . \mathrm{kg}^{-1}$ plus atropine $0.02 \mathrm{mg} \cdot \mathrm{kg}^{-1}$, and awake extubation after fulfilment of extubation criteria. After awakening from anesthesia, $\mathrm{VAS}^{0}$ were assessed before patients transfer to PACU.

Postoperatively at PACU, patients were observed for hemodynamics, pain assessment by VAS for one hour. At surgical ward, patients received paracetamol $1 \mathrm{~g} / 8 \mathrm{~h}$., ringer acetate $4 \mathrm{ml} \cdot \mathrm{kg}^{-1} \cdot \mathrm{h}^{-1}$ till starting oral intake. Rescue analgesia based on pethidine $0.5 \mathrm{mg} . \mathrm{kg}^{-1} \mathrm{IV}$ when VAS $>4$. Data recorded by trained nurse included VAS, HR, MBP, $\mathrm{SPO}_{2}$ at $1 \mathrm{H}, 2 \mathrm{H}, 4 \mathrm{H}, 6 \mathrm{H}, 12 \mathrm{H}, 24$ hours postoperatively, total pethidine intake, first time to pethidine intake and incidence of nausea, vomiting within first 24 hours. Any other side effects, complications or patient's complaints related to study medication were reported and documented.

In this trial, we hypothesized that subcutaneous ketamine infiltration may control postoperative pain better than intravenous route with stable hemodynamics. Primary outcome objective included pain assessment by VAS score for 24 hours postoperatively, while secondary outcomes included hemodynamics data, rescue analgesia consumption total dose, first time to request analgesia and incidence of PONV for 24 hours postoperatively.

\section{Sample size statistical analysis and data collection:}

Sample size was calculated using $G^{*}$ Power for windows (ver. 3.0.10). Pain assessment by VAS was used as the primary effect. One tailed $t$ test for difference between two independent means was the computed statistical test. A 25\% reduction of the VAS in the first hour by, retrieved form the results of a previous study ${ }^{(9)}$, was considered as an acceptable effect size. Targeting a study power of $90 \%$ with an alpha error of 0.05 , a total sample size was 23 patients were required for each group. A total number of 60 patients in both groups were recruited to compensate for dropouts. P-value was considered significant if less than 0.05 .

Perioperative data were tabulated and analyzed using IBM SPSS software version 22. Continuous data were presented as mean and SD. Nominal and categorical data were presented as numbers and percentages. Independent sample T test, MannWhitney test, chi square test were utilized to detect statistical differences between the studied groups. P value less than 0.05 was considered significant.

\section{RESULTS}

In this study, 61 patients matched eligibility criteria. One patient was excluded due to patient's refusal to participate in our research (Figure 1). 


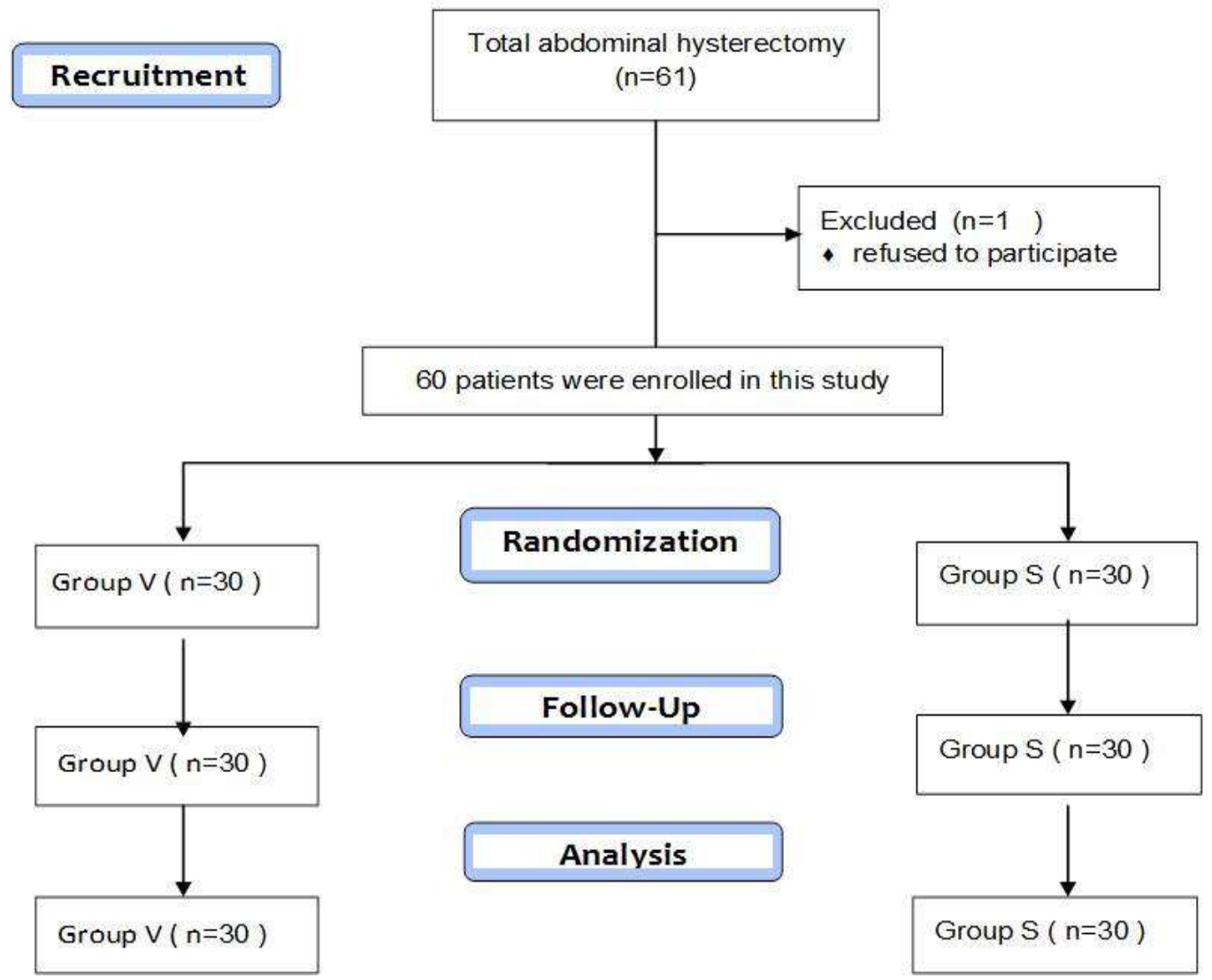

Figure (1): CONSORT flow diagram for the study

Perioperative characteristics of the included patients are presented in table (1), with significant difference in the first time to pethidine intake. None of the patients showed any side effects or un-anticipated complications related to study medication.

Table 1: Perioperative characteristics of the two studied groups. Data are presented as mean \pm SD and absolute number (\%)

\begin{tabular}{|l|l|l|l|}
\hline & Group v & Group s & P value \\
\hline Age (years) & $48.03 \pm 9.23$ & $50.83 \pm 7.16$ & 0.195 \\
\hline $\begin{array}{l}\text { BMI (weight in kg/height in } \\
\text { meter }^{2} \text { ) }\end{array}$ & $26.45 \pm 3.21$ & $25.59 \pm 3.58$ & 0.334 \\
\hline Surgical time (min) & $100.50 \pm 15.99$ & $102.50 \pm 18.37$ & 0.655 \\
\hline First time to pethidine (hours) & $1.50 \pm 0.93$ & $2.36 \pm 1.27$ & $0.004 *$ \\
\hline Total pethidine dose (mg) & $72.00 \pm 27.84$ & $59.33 \pm 23.18$ & 0.061 \\
\hline PONV & $7(23.3 \%)$ & $5(16.6 \%)$ & 0.519 \\
\hline $\begin{array}{l}\text { BMI: body mass index, PONV: postoperative nausea and vomiting. } \\
\text { * Significant difference }\end{array}$
\end{tabular}

Intraoperative hemodynamics, heart rate (HR) and mean blood pressure (MBP), of the included patients are presented in figure (2), postoperative hemodynamics (HR, MBP) of the included patients are presented in figure (3), with no significant difference among the included patients of both groups. 


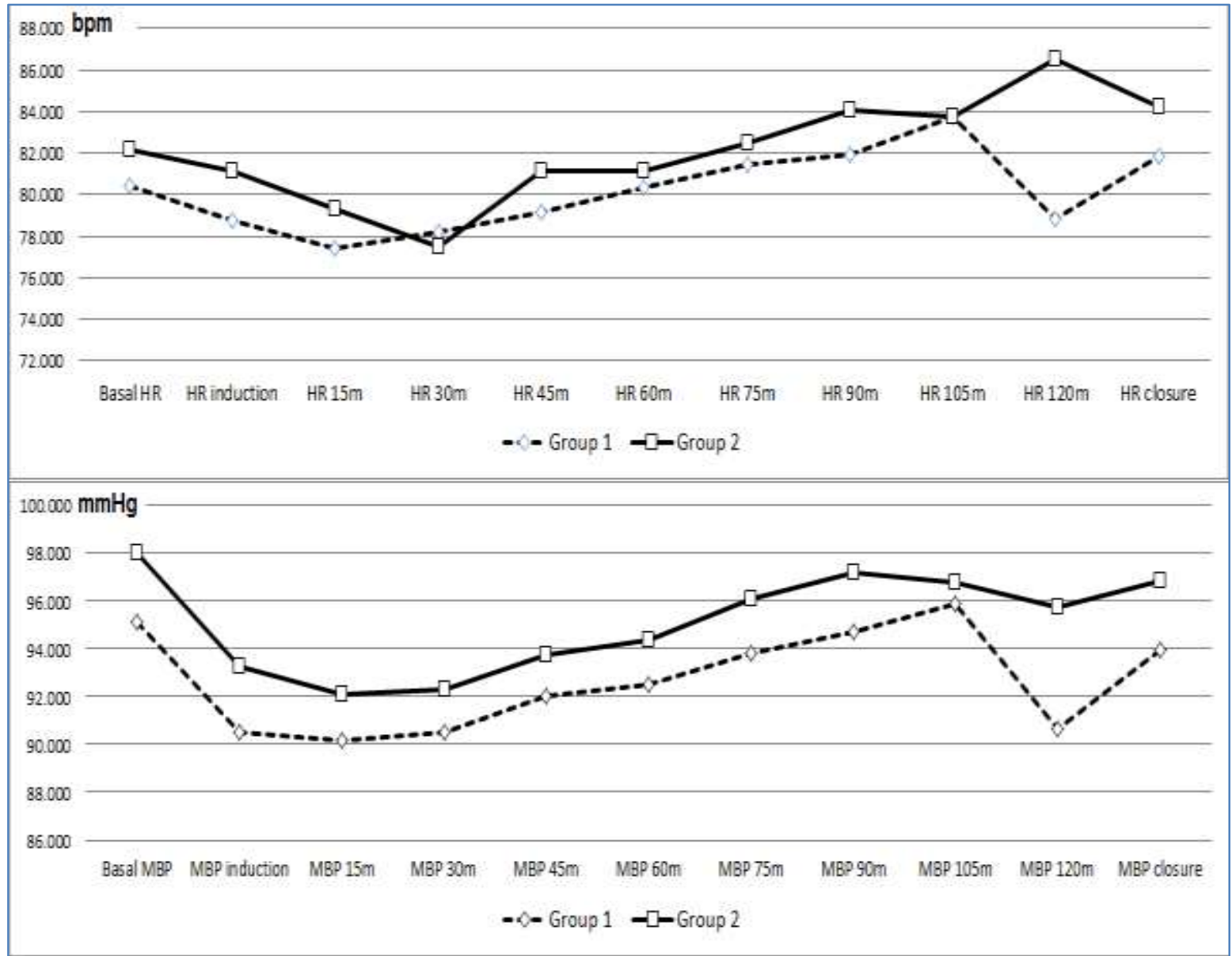

Figure (2): Intraoperative hemodynamics data; heart rate (HR) and mean blood pressure (MBP) of the included patients; Group 1(V group) and Group 2 (S group).

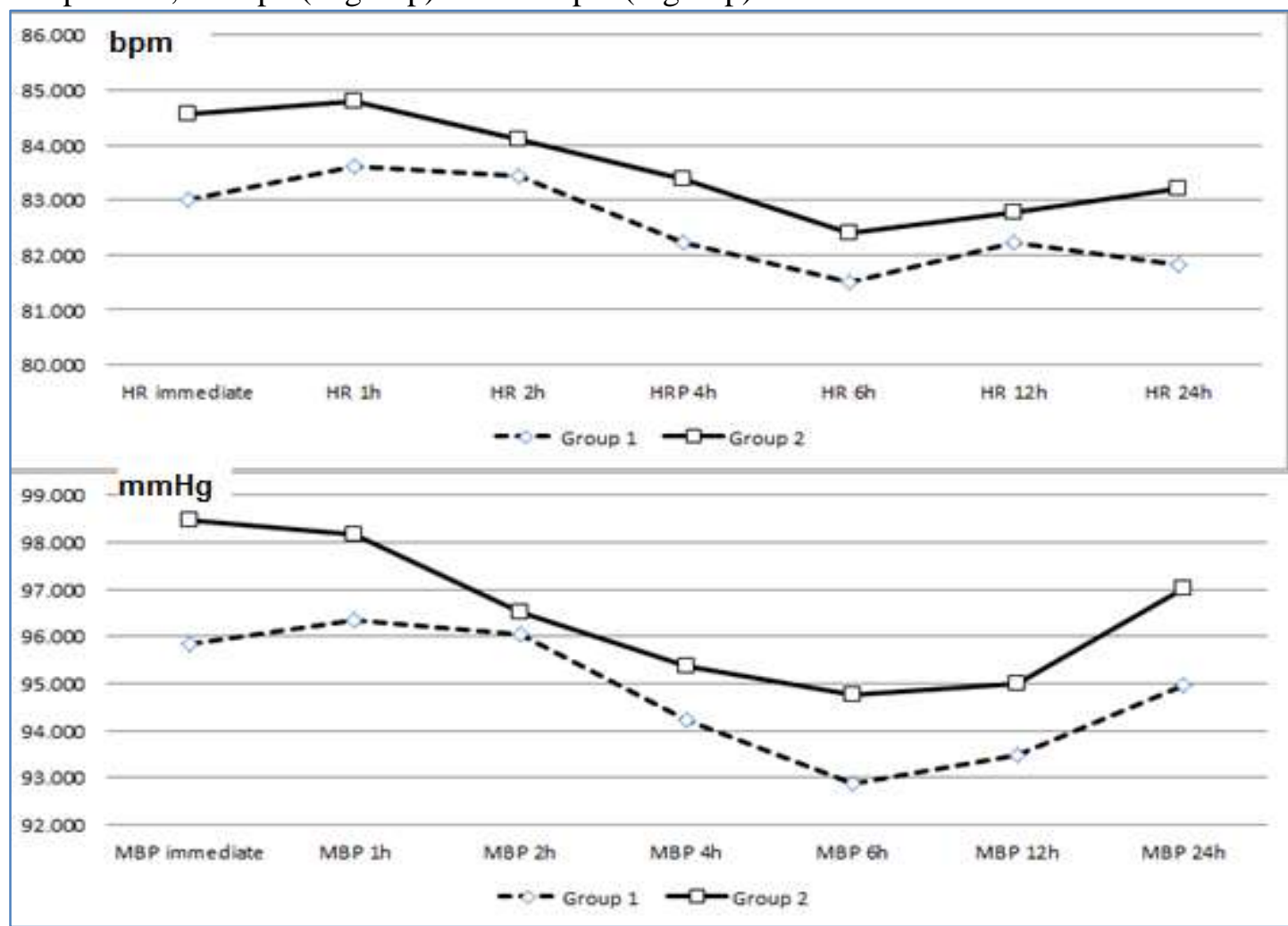

Figure (3): Postoperative hemodynamics data (HR, MBP) of the included patients; Group 1 (V group) and Group 2 (S group).

VAS score for postoperative pain assessment during first 24 hours among the included patients is presented in (table 2), with significant difference as: $\mathrm{VAS}^{0}, \mathrm{VAS}^{1}$, and $\mathrm{VAS}^{2}$, while other time points were comparable. 
Table (2): VAS score during the first postoperative 24 hours. Data are presented as mean \pm SD.

\begin{tabular}{|l|r|r|c|}
\hline & Group V & Group S & P value \\
\hline VAS $^{0}$ & $2.40 \pm 0.62$ & $1.96 \pm 0.92$ & $0.038^{*}$ \\
\hline $\mathrm{VAS}^{1}$ & $3.96 \pm 1.21$ & $2.86 \pm 0.89$ & $0.001^{*}$ \\
\hline $\mathrm{VAS}^{2}$ & $4.00 \pm 0.94$ & $3.33 \pm 0.88$ & $0.007 *$ \\
\hline $\mathrm{VAS}^{4}$ & $3.33 \pm 0.75$ & $3.30 \pm 0.79$ & 0.869 \\
\hline $\mathrm{VAS}^{6}$ & $2.26 \pm 0.78$ & $1.96 \pm 0.61$ & 0.105 \\
\hline $\mathrm{VAS}^{12}$ & $1.46 \pm 0.73$ & $1.26 \pm 0.78$ & 0.311 \\
\hline $\mathrm{VAS}^{24}$ & $1.23 \pm 0.62$ & $1.26 \pm 0.63$ & 0.839 \\
\hline $\begin{array}{l}\text { VAS: visual analogue score. } \\
\text { * Significant difference }\end{array}$
\end{tabular}

\section{DISCUSSION}

In this prospective, controlled, double blinded trial, we compared preemptive use of ketamine at analgesic dose by two different routes of administration either intravenous injection or subcutaneous infilteration at incisional site. 60 patients planned for total abdominal hysterectomy were enrolled. Postoperative pain assessment using VAS score was adapted as primary outcome objective of this study.

We found that subcutaneous infiltration of ketamine at incisional site provided better analgesia at early postoperative times presented by lower VAS score within the first two postoperative hours and delayed time of rescue analgesia request. While we could not find any significant difference between both routes regarding VAS score later than the first two hours, hemodynamics, PONV or other side effects and complications.

Ketamine is a NMDA-receptor antagonist with anti-inflammatory effect ${ }^{(7)}$. Its analgesic effects is related to dual mechanism of actions, central and local effects. It produces central desensitization of pain pathway, reducing central hyperalgesia specially when used preemptively ${ }^{(8)}$. Moreover, many studies described the value of ketamine use in reduction of chronic post-surgical pain ${ }^{(10,11)}$. Also, it has been shown that ketamine have a local anesthetic effect mediated by a depression of sodium channel function. This effect is augmented by anti-proinflammatory action. It interacts with inflammatory cells recruitment, cytokines production, and inflammatory mediator regulation ${ }^{(12)}$.

Preemptive use of ketamine is a wellestablished concept, many trial and meta-analysis documented it's analgesic efficacy through reduction of pain score or consumption of rescue analgesia ${ }^{(13,14)}$. Preemptive analgesic efficacy of ketamine was proved in different surgical techniques, either open surgery $^{(15,16)}$ or laparoscopic one ${ }^{(17,18)}$. Also, by different route of administration, either intravenous ${ }^{(19)}$, subcutaneous ${ }^{(7,16)}$, inhalational nasally ${ }^{(20)}$ or regional nerve blocks ${ }^{(21)}$. However earlier limited studies provided no beneficial analgesic effect ${ }^{1(22,23)}$.
Many trials compared the analgesic efficacy of ketamine with local anesthetics and found no superiority of local anesthetic's infiltration ${ }^{(12)}$. They concluded that ketamine may provide better systemic effect as analgesia and anti-inflammatory action, while local anesthetics is restricted to block of nerve impulse conduction by inhibition of sodium channels at the nerve endings and along the axon only ${ }^{(24)}$.

According to our result, pre incisional subcutaneous ketamine infiltration at incision site had better analgesic profile at early postoperative period. We can attribute this results to dual mechanism of action, central and local analgesic effects. Also, the pharmacokinetics and pharmacodynamics characters of ketamine have a role. Ketamine is highly lipid soluble but has low protein-binding ability. Ketamine is mostly metabolised in the liver (80\%) into norketamine $^{(25)}$. Peak levels of norketamine appear within the blood around 30 minutes after intravenous administration $^{(26)}$. Also, at anesthetic dose and after subcutaneous injection, the effect start within 10-15 min and last for 30-120 min. while after intravenous injection, the effect start within 1-2 min and last for 20-60 min. This character shows nearly double of the duration effect ${ }^{(27,28)}$. This duration effect can be prolonged to analgesic action specially when synergized with other opioid and non-opioid analgesics ${ }^{(28)}$. So, we can consize that subcutaneous route owing to delayed absorption and blood bioavailability have longer duration of action than intravenous route specially in our group of patients with BMI at overweight range and excessed accumulation of fat.

Our results are matching with Safavi et al. (16) in their trial on open cholecystectomy patients. They proved comparability of both routes with better analgesia in subcutaneous infiltration when increased ketamine dose to $2 \mathrm{mg} \cdot \mathrm{kg}^{-1}$. While in adenotonsillectomy surgery, khademi et al. (29) documented better analgesia with peritonsillar infiltration rather than intravenous ketamine use. Meanwhile, Dal et al. ${ }^{(30)}$ found no difference between both routes. 
Honarmand et al. (31) approved better analgesia with intravenous route on patients underwent appendectomy surgeries. They had lower VAS score and less rescue analgesia consumption. We can attribute this to simplicity of procedure and shorter surgery time. This made the bioavailability of ketamine in the blood with intravenous route more rapid with better analgesia.

All these conflicting results are probably due to heterogeneity of the studies, different procedures, techniques, ketamine dosing and intake time, age and gender group. So, we need further studies in such group of patients using multiple doses to evaluate efficacy of each route of administration.

Our results found no significant difference among both studied group regarding intra- and postoperative hemodynamics, oxygen saturation and PONV. Also, no complications related to study medication had been detected.

Limitation of our study includes: overweight of patients, which may not represent population especially in highly lipid soluble used drug. Pain assessment by VAS score only without using stress markers, which may give more reliable results. Needs for further studies with titrating rising doses may prolong postoperative analgesia and reflect more obvious results between both routes.

\section{CONCLUSION}

Preemptive subcutaneous ketamine at incisional site provided more analgesic effect in early postoperative period rather than intravenous administration in total abdominal hysterectomy patients, without significant difference in hemodynamics and PONV.

\section{ACKNOWLEDGMENT}

For staff nurses in operating room and gynecological ward, Mansoura University Hospital, Egypt, who provided high quality perioperative care for our patients.

\section{REFERENCES}

1. Farzi F, Naderi B, Mirmansouri A et al. (2016): Postoperative pain after abdominal hysterectomy: A randomized, double-blind controlled trial comparing the effects of tramadol and gabapentin as premedication. Anesth Pain Med.,6 (1): 32-6.

2. Gan T (2017): Poorly controlled postoperative pain: prevalence, consequences and prevention. J Pain Res., 10: 2287-98.

3. Oh S, Lee I, Lim B et al. (2019): Comparison of the analgesic effect of sufentanil versus fentanyl in intravenous patient-controlled analgesia after total laparoscopic hysterectomy: A randomized double-blind prospective study. Int J Med Sci., 16(11):1439-46.

4. Mathew P, Aggarwal N, Kumari K et al. (2019): Quality of recovery and analgesia after total abdominal hysterectomy under general anesthesia: A randomized controlled trial of TAP block vs epidural analgesia vs parenteral medications. Journal of Anaesthesiology, Clinical Pharmacology, 35(2):170-5.

5. Arbela R, Stanleigha J, Ioscovich A (2013): Pain management following abdominal hysterectomy: Novel approaches and review of the literature. J Clin Gynecol Obstet., 2(2):51-5.

6. Jain R and Kochhar N (2017): Influence of difference in timing of perioperative administration of low-dose ketamine on postoperative analgesia. Anesth Essays Res., 11:406-10.

7. Behaeen $\mathrm{K}$, Soltanzadeh $\mathrm{M}$, Nesioonpour $\mathrm{S}$ et al. (2014): Analgesic effect of low dose subcutaneous ketamine administration before and after cesarean section. Iran Red Crescent Med J., 16(3): 1550-6.

8. Sleigh J, Harvey M, Voss L et al. (2014): Ketaminemore mechanisms of action than just NMDA blockade. Trends Anaesth Crit Care, 4 (2):76-81.

9. Süner ZC, Kalaycı D, Sen Ö et al. (2019): Postoperative analgesia after total abdominal hysterectomy:Is the transversus abdominis plane block effective?. Niger J Clin Pract., 22:478-84.

10. Gorlin A, Rosenfeld D, Ramakrishna H (2016): Intravenous sub-anesthetic ketamine for perioperative analgesia. J Anaesthesiol Clin Pharmacol., 32(2):160-7.

11. McNicol E, Schumann R, Haroutounian S (2014): A systematic review and meta-analysis of ketamine for the prevention of persistent post-surgical pain. Acta Anaesthesiol Scand., 58(10):1199-213.

12. Abdallah N, Salama A, Ellithy A (2017): Effects of preincisional analgesia with surgical site infiltration of ketamine or levobupivacaine in patients undergoing abdominal hysterectomy under general anesthesia; A randomized double blind study. Saudi J Anaesth., 11:267-72.

13. García J, Moral J, Salazar A et al. (2018): Effects of ketamine on postoperative pain after remifentanil-based anesthesia for major and minor surgery in adults: A systematic review and meta-analysis. Front. Pharmacol., $9: 9-21$.

14. Yang $\mathrm{L}$, Zhang J, Zhang $Z$ et al. (2014): Preemptive analgesia effects of ketamine in patients undergoing surgery. A meta-analysis. Acta Cir Bras. , 29 (12):81925.

15. Parikh B, Maliwad J, Shah V (2011): Preventive analgesia: Effect of small dose of ketamine on morphine requirement after renal surgery. J Anaesthesiol Clin Pharmacol., 27(4):485-8.

16. Safavi M, Honarmand A, Nematollahy $Z$ (2011): Preincisional analgesia with intravenous or subcutaneous infiltration of ketamine reduces postoperative pain in patients after open cholecystectomy: a randomized, double-blind, placebo-controlled study. Pain Med., 12(9):1418-26.

17. Lin H, Jia D (2016): Effect of preemptive ketamine administration on postoperative visceral pain after gynecological laparoscopic surgery. J. Huazhong Univ Sci Technolog Med Sci., 36(4): 584-87.

18. Singh H, Kundra S, Singh R et al. (2013): Preemptive analgesia with ketamine for laparoscopic cholecystectomy. J Anaesthesiol Clin Pharmacol., 29:478-84.

19. Chen J, Hu W, Li S et al. (2020): The efficacy of ketamine in total knee arthroplasty: a randomized controlled trial protocol, Medicine, 99:(24)206-45. 
20. Abdel-Ghaffar H, Abdel-Wahab A, Roushdy M et al. (2019): Preemptive nebulized ketamine for pain control after tonsillectomy in children: randomized controlled trial. Rev. Bras. Anestesiol., 69(4): 350-7.

21. Krishna G, Khanna S, Jaishree $\mathbf{S}$ (2020): Review of adjuvants to local anesthetics in peripheral nerve blocks: Current and future trends. Saudi J Anaesth., 14:77-84.

22. Karaman S, Kocabas S, Zincircioglu C et al. (2006): Has ketamine preemptive analgesic effect in patients undergoing abdominal hysterectomy? Agri., 18(3):3644.

23. Nistal-Nuño B, Freire-Vila E, Castro-Seoane F et al. (2014): Preoperative low-dose ketamine has no preemptive analgesic effect in opioid-naïve patients undergoing colon surgery when nitrous oxide is used - a randomized study. Food research, 3:226.

24. Heavner J (2007): Local anesthetics. Curr Opin Anaesthesiol., 20:336-42.

25. Abdollahpour A, Saffarieh E, Zoroufchi B (2020): A review on the recent application of ketamine in management of anesthesia, pain, and health care. J Family Med Prim Care, 9:1317-24.

26. Luft A, Mendes F (2005): Low $S(+)$ ketamine doses: a review. Rev. Bras. Anestesiol., 55 (4):460-9.
27. Jasper K, Erik O, Thomas K et al. (2020): For the ketamine pharmacokinetic study group; ketamine pharmacokinetics: A systematic review of the literature, meta-analysis, and population analysis. Anesthesiology, 133:1192-213.

28. Peltoniemi M, Hagelberg N, Olkkola K et al. (2016): Ketamine: A review of clinical pharmacokinetics and pharmacodynamics in anesthesia and pain therapy. Clin Pharmacokinet, 55:1059-77.

29. Khademi S, Ghaffarpasand F, Heiran H et al. (2011): Intravenous and peritonsillar infiltration of ketamine for postoperative pain after adenotonsillectomy: a randomized placebo-controlled clinical trial. Med Princ Pract., 20(5):433-7.

30. Dal D, Celebi N, Elvan E et al. (2007): The efficacy of intravenous or peritonsillar infiltration of ketamine for postoperative pain relief in children following adenotonsillectomy. Paediatr Anaesth., 17(3):263-9.

31. Honarmand A, Safavi M, Karaky H (2012): Preincisional administration of intravenous or subcutaneous infiltration of low-dose ketamine suppresses postoperative pain after appendectomy. Journal of Pain Research, 5: 1-6. 